\title{
Zeolite Crystal Growth Analysis by Dissolution
}

Krassimir N. Bozhilov ${ }^{1}$ and Valentin Valtchev ${ }^{2}$

1. Central Facility for Advanced Microscopy and Microanalysis, University of California, Riverside, CA, USA

2. Laboratoire Catalyse et Spectrochimie (LCS), Université de Caen - CNRS, Caen, France.

* Corresponding author: bozhilov@ucr.edu

Zeolites are widely used in many commercial processes, mostly as catalysts or adsorbents. Understanding their crystal growth mechanism is a key to control their properties and design the best materials for their ever increasing uses [1]. Crystal growth of zeolites has been extensively studied and the major aspects are well known, although still great uncertainty exists concerning the mechanism of nucleation and the transition from nucleation to growth stage [2].

By applying detailed structural and chemical analysis by analytical scanning and transmission electron microscopy aided by focused ion beam sample preparation we have studied time series dissolution experiments of silica-based MFI-and MOR-type zeolite crystals in ammonium fluoride medium, which allowed us to extrapolate and elucidate the possible mechanism of crystal growth and nucleation [3]. The dissolution profiles in these commercially relevant zeolite crystals reveal sub-structure that in turn allows information about the abundance and topology of initial nucleation sites to be extracted.

Dissolution of silicates in water has been studied in detail $[4,5]$ and it is established that the sites of bond breaking in the rate-determining step of dissolution are the weakest bonds. In silica-based zeolites only $\mathrm{Si}-\mathrm{O}$ and $\mathrm{Si}-\mathrm{OH}$ bonds exist, as a consequence the weakest bonds are the bonds under structural stress caused by defects. Since dissolution could be regarded as reciprocal to growth in temporal aspect, the weak bonds that break first will be corresponding to the weak bonds that form last during crystal growth. Following this approach, it is considered that domains corresponding to stable viable nuclei should dissolve last, allowing to shed light on the hydrothermal crystal growth mechanism of zeolites by ex-situ methods and reveal information about the critical steps in their nucleation and growth.

Application of non-selective dissolution of zeolite by fluoride etching method [6] readily removes framework cations and produces crystals with composition similar to the parent material. Our study reveals that dissolution starts at defect sites with strained Si-O bonding such as point defects, dislocations, twin boundaries, low-angle grain boundaries and proceeds by expanding around initially formed dissolution pits. The dissolution then advances parallel to low-index atomic planes with small reticular density in a reciprocity to the Bravais-Donnay-Harker growth law. The periphery of the studied zeolite crystals appears to be the most stable part [figure 1]. It was established that zeolite crystals have distinct mosaic structure which can evolve during the process of growth and it can reflect the changing conditions of growth and thus marking the different growth stages.

Our interpretation is that growth proceeds by aggregation of viable nuclei with zeolite crystalline structure with sizes in the range of $10-20 \mathrm{~nm}$. The interfaces of the connecting nanoparticles confine and seal defects such as lattice and bond distortions, and point defects. The central parts of the crystals form fast due to supersaturation and large number of nuclei present, which tends to concentrate high level of defects. Following this nucleation stage growth proceeds by addition of both small crystalline slabs as well as 
oligomers from the solution. During this stage growth and dissolution are competing processes resulting in the final equilibrium crystal form faceted by low index slow growing faces.

This study shows that detailed analysis of the domain structure developed during dissolution can be used successfully as indirect yet effective method for advancing our understanding of zeolite growth mechanism and consequently lead to rational design of superior catalysts and adsorbents. Further, more general implication of our observations concerns the mosaic structure in crystals that has been known for long time [7], but no direct correlation of its effect on the growth mechanism of macrocrystals has been demonstrated up till now.

\section{References:}

[1] J Čejka et al., in "Zeolites and Catalysis: Synthesis, Reactions and Applications” (Wiley-VCH, Weinheim).

[2] A Corma and ME Davis, ChemPhysChem 5 (2004), p. 304.

[3] JR Agger et al., J. Am. Chem. Soc. 125 (2003), p. 830.

[4] FK Grundwell, Hydrometallurgy 139 (2013), p. 132.

[5] FK Grundwell, Hydrometallurgy 149 (2014), p. 265.

[6] Z Qin,et al., Chem. Mater. 25 (2013), p. 2759.

[7] CG Darwin and CG Phil. Mag. 43 (1922), p. 800.

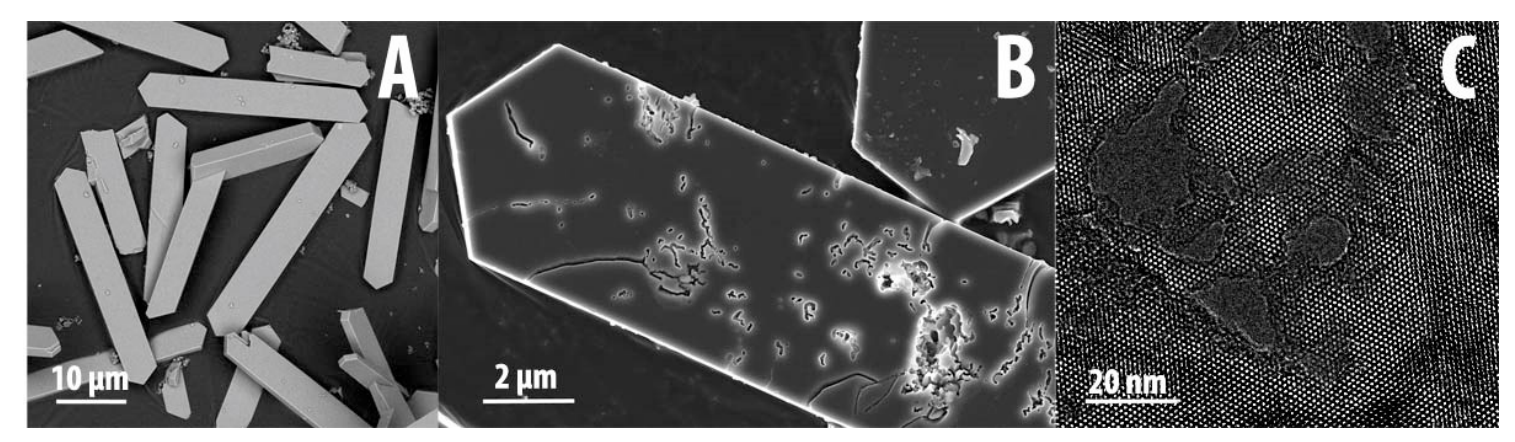

Figure 1. The consecutive stages of dissolution of ZSM-5 crystals are shown. The BSE image in (A) demonstrates the morphology of un-treated ZSM-5 crystals. A complex pore system has developed after 30 min of fluoride-etching, SE image (B). The HRTEM image of the zeolite crystal lattice in (C) reveals the crystal domains and extensive pores formed after 60 min of treatment. 